\title{
Surface energy and relaxation in boron carbide (10ī1) from first principles
}

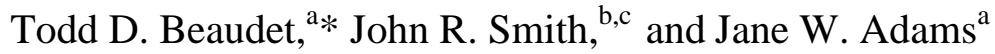 \\ ${ }^{a}$ U.S. Army Research Lab, Aberdeen Proving Ground, MD 21005 \\ ${ }^{b}$ Dept. of Materials Science and Engineering, University of Michigan, Ann Arbor, MI 48109 \\ ${ }^{c}$ Dept. of Mechanical Engineering, Johns Hopkins University, Baltimore, MD 21218
}

\begin{abstract}
The surface energy of the boron carbide polytype $B_{11} C_{p}(C B C)$ for planar separations along $\{10 \overline{1} 1\}$ was determined to be $3.21 \mathrm{~J} / \mathrm{m}^{2}$ via first-principles density-functional computations. Surface atomic relaxations are relatively large, thereby lowering the surface energy significantly. The icosahedra are not intact on the surface, i.e., severed polyhedra are the lowest energy surface configuration. Good agreement was found with an experimental average fracture surface energy.
\end{abstract}

Keywords: E. Density functional theory; C. Surface energy; C. Work of separation; D. Fracture

\section{Introduction}

Boron carbide materials have interesting, technologically important properties [1-9]. Their high hardness ( 25 to $35 \mathrm{GPa}$ - next only to diamond and cubic boron nitride), high elastic modulus $(450 \mathrm{GPa})$, high flexural strength $(350-500 \mathrm{MPa})$, high melting point $\left(2450{ }^{\circ} \mathrm{C}\right)$ and low density $\left(2.52 \mathrm{~g} / \mathrm{cm}^{3}\right)$ are of interest for applications to lightweight armor and grinding materials. A high capture cross section for neutrons is important for neutron absorption in reactors and a substantial Seebeck coefficient up to high temperatures finds application in thermoelectrics $[1-3,10]$.

Here a first-principles, density functional theory (DFT) [11-12] investigation of some of the surface properties of boron carbide is presented. The $\{10 \overline{1} 1\}$ surface energy $\sigma$ is given by

$$
\sigma=W_{\text {sep }} / 2=\left(U_{s}-U_{0}\right) / 2 A
$$

where $U_{0}$ is the total energy of the bulk crystalline boron carbide, $U_{s}$ is the total energy after the bulk crystal is fully separated along a $\{10 \overline{1} 1\}$ plane, $A$ is the cross-sectional area of the crystal, and $W_{\text {sep }}$ is the work of separation per area $A$.

The boron carbide bulk atomic structure consists of 12-atom icosahedrons cross-linked by 3atom chains, as shown in Fig. 1. Within the icosahedra, 6 atoms are in polar sites and 6 atoms are in equatorial sites. In addition to the intra-icosahedral bonds, the polar atoms are linked to other icosahedra by inter-icosahedral bonds and the equatorial atoms are linked by the 3 atom chains [13]. The stoichiometry of boron carbide, $\mathrm{B}_{\mathrm{x}} \mathrm{C}$, can vary through the range $4.3 \leq \mathrm{x} \leq 10.8$. For a given stoichiometry, there can be compositional variation cell to cell, such as $\mathrm{B}_{12}$ or $\mathrm{B}_{11} \mathrm{C}$, and the chain sites can be C-B-C, C-B-B or B-vacancy-B, depending on how carbon-rich the

Corresponding author. Tel.: +1 410306 4963; fax: +1 410306 0829; e-mail:

todd.d.beaudet.ctr@mail.mil 
system is [1]. Each composition is called a polytype. For these initial boron carbide surface energy computations it is necessary to choose a specific polytype to study. It has been found [1] through IR phonon spectral analysis that, in the $\mathrm{C}$-rich limit, the $\mathrm{B}_{11} \mathrm{C}(\mathrm{CBC})$ polytype makes up $91 \%$ of boron carbide. The $\mathrm{B}_{11} \mathrm{C}_{\mathrm{p}}(\mathrm{CBC})$ polytype (subscript $\mathrm{p}$ refers to a polar site and the $\mathrm{CBC}$ in parenthesis indicates the chain composition) also exhibits the maximum yield strength under uniaxial compression $(\approx 140 \mathrm{GPa})$ [14]. For these reasons the polytype $\mathrm{B}_{11} \mathrm{C}_{\mathrm{p}}(\mathrm{CBC})$ is chosen for the initial surface energy study presented here.

\section{Computational Methods}

The total energies $U_{0}$ and $U_{s}$ of Eq. (1) are computed via DFT. The DFT method employed here uses the generalized gradient approximation (GGA) exchange-correlation functional of Perdew, Burke, and Ernzerhof (PBE) [15-16]. These calculations were performed using the CP2K code [17] which uses the Quick-Step method as described by VandeVondele et al. [18]. This methodology uses a Gaussian basis to describe the single-body functions (i.e., molecular orbitals) and a multi-grid plane wave basis for the electron density. Goedecker, Teter, and Hutter (GTH) [19-20] pseudopotentials are used to treat the $1 \mathrm{~s}^{2}$ electronic cores of boron and carbon. These separable dual-space Gaussian-type pseudopotentials are optimized for PBE by Krack [21]. A Gaussian basis set with triple-zeta and two valence polarization functions is used for the single body orbitals, as is a plane wave cutoff of $3810 \mathrm{eV}$ for the electron density. Energy selfconsistence was converged to $2.72 \times 10^{-4} \mathrm{eV}$ and forces were converged to $0.0231 \mathrm{eV} / \AA$. Results are given for the gamma point where the simulation cell contains 180 atoms and lattice vectors are nearly orthogonal and approximately $10 \AA$ in length prior to separation. All calculations use periodic boundary conditions. The initial bulk structure was relaxed so that all stress tensor elements were below $10 \mathrm{MPa}(100 \mathrm{bar})$. Using this level of theory gives lattice parameters which are in good agreement with experiment at the carbon rich limit which is near $\mathrm{B}_{4} \mathrm{C}$ stoichiometry. The lattice parameters $a$ and $c$ were found to be $5.599 \AA$ and $12.055 \AA$, within $0.6 \%$ and $0.25 \%$ of experimental values respectively $[1,22]$. It should be noted that hexagonal symmetry is broken due to the polar carbon in the $\mathrm{B}_{11} \mathrm{C}_{\mathrm{p}}(\mathrm{CBC})$ polytype resulting in the value of $a$ varying by $+0.005 /-0.009$ about the average value of $5.599 \AA$ as well as a slight $2.11^{\circ}$ deflection of the $c$-axis with respect to the basal plane normal; this is expected.

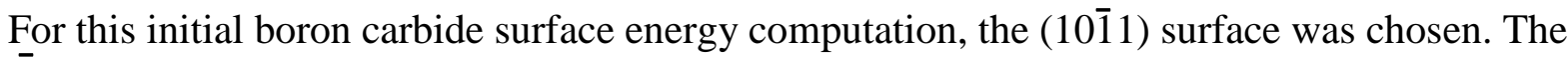
$\{10 \overline{1} 1\}$ family of planes have the lowest interfacial unit cell area, the highest density of atoms within plane, and correspondingly the smallest number of bonds to be broken to create the free surfaces.

Determining the surfaces with the lowest surface energy within the $\{10 \overline{1} 1\}$ family of planes is more challenging. Since the $\mathrm{B}_{11} \mathrm{C}_{\mathrm{p}}(\mathrm{CBC})$ polytype has 15 atoms per primitive cell and relatively low monoclinic Pm symmetry due to the polar carbon, it is not clear a priori where within the family of $\{10 \overline{1} 1\}$ planes the lowest $W_{\text {sep }}$ is likely to occur. It is necessary then to investigate a number of $\{10 \overline{1} 1\}$ separation planes across the unit cell. It will be seen below that $13\{10 \overline{1} 1\}$ separation plane locales are treated. However, for a given separation plane locale, it is not clear which surface structure will be most energetically favorable because there are atoms which are relatively close to a separation plane. To investigate this, atoms within $0.25 \AA$ on either side of the separation plane are tested as to which side or surface of the separation they will reside on. 
This means that a relatively large number of surfaces must be investigated, in combinatorial fashion, to determine the lowest $W_{\text {sep }}$ for the $\{10 \overline{1} 1\}$ family of planes.

For each separation plane studied, the bulk model was cleaved in rigid fashion to $20.0 \AA$ such that bonds are broken across the interface while bonds within each solid remain frozen at their bulk values. Subsequently, all atoms positions are allowed to relax through geometry optimization. The final energy is the separated crystal energy $U_{s}$. The surface energy $\sigma$ and work of separation $W_{\text {sep }}$ can then be computed via Eq. (1). Equivalently, the final $W_{\text {sep }}$ can be computed as a rigid separation energy minus the surface relaxation energy. In this way the rigid separation energy and the surface relaxation energy can be understood as two separate energy components of $W_{\text {sep }}$. The surface relaxation energy quantifies the restructuring from the rigid reference.

\section{Results}

A summary of the lowest $W_{\text {sep }}$ results for each separation plane is shown in Fig. 1. The reference bulk structure is separated along planes perpendicular to the paper, shown as dotted lines, passing through the corresponding point in the $W_{\text {sep }}$ plot. The $W_{\text {sep }}$ values shown in Fig. 1 and listed in the first column of Table 1 are in the range $6.42-8.14 \mathrm{~J} / \mathrm{m}^{2}$. The lowest surface energy determines the equilibrium surface. The lowest energy surface is then formed with a $W_{\text {sep }}=6.42 \mathrm{~J} / \mathrm{m}^{2}$ or a surface energy $\sigma=3.21 \mathrm{~J} / \mathrm{m}^{2}$. This lowest surface energy is obtained via the planar separation at (a) in Figs. 1 and 2.

A table of bulk bonds broken to form the surfaces is found in Table I. As discussed above, a rigid separation of the bulk solid is performed first with a subsequent relaxation. This allows the breakdown of $W_{\text {sep }}$ into a rigid separation energy plus a relaxation energy for an enhanced understanding.

The rigid separation energies are shown in column 2 of Table I. Table I also tabulates the number of each type of bond broken with respect to each rigid fracture (columns 4-9). For a rigid separation, one can attempt to connect with the substantial literature on relative bonds stiffnesses in boron carbide (for a review, see Domnich et al. [13] especially Section IV, p. 3614). This literature suggests that bond stiffnesses in decreasing order are intra-chain, chainicosahedra, inter-icosahedra, and intra-icosahedra. One must bear in mind that the prevailing literature relates to bond stiffnesses while Table I relates to separation energies. Generally though, stiffer bonds are associated with higher bond energies. In this regard, it can be seen somewhat qualitatively from Table I that the intra-icosahedra bonds appear lower in energy than the non-intra-icosahedra bonds. For example, the third and fourth rows of Table I show only intra-icosahedra bonds broken (columns 4-7 are zero) with each separation breaking 40 such bonds. The last two rows show only non-intra-icosahedra bonds broken (columns 8-9 are zero) with 16 broken in each case. Still, a more comprehensive analysis is desirable.

TABLE I. Components of the $\mathrm{B}_{11} \mathrm{C}_{\mathrm{p}}(\mathrm{CBC}) W_{\text {sep }}$ values plotted in Fig. 1. Subscripts c and $\mathrm{i}$ indicate chain and icosahedra atoms respectively. Bonds involving icosahedra are distinguished as inter- or intra-icosahedra bonds. 


\begin{tabular}{|c|c|c|c|c|c|c|c|c|}
\hline \multirow{2}{*}{$\begin{array}{l}W_{s e p} \\
\left(\mathrm{~J} / \mathrm{m}^{2}\right)\end{array}$} & \multirow{2}{*}{$\begin{array}{c}\text { Rigid } \\
\text { Separation } \\
\text { Energy } \\
\left(\mathrm{J} / \mathrm{m}^{2}\right)\end{array}$} & \multirow{2}{*}{$\begin{array}{c}\text { Surface } \\
\text { Relaxation } \\
\text { Energy } \\
\left(\mathrm{J} / \mathrm{m}^{2}\right)\end{array}$} & \multicolumn{6}{|c|}{ Bonds Broken in Rigid Separation } \\
\hline & & & $\mathrm{C}_{\mathrm{c}}-\mathrm{B}_{\mathrm{c}}$ & $\mathrm{C}_{\mathrm{c}}-\mathrm{B}_{\mathrm{i}}$ & $\begin{array}{c}\text { Inter } \\
C_{i}-B_{i}\end{array}$ & $\begin{array}{c}\text { Inter } \\
B_{i}-B_{i}\end{array}$ & $\begin{array}{c}\text { Intra } \\
C_{i}-B_{i}\end{array}$ & $\begin{array}{c}\text { Intra } \\
\mathrm{B}_{\mathrm{i}}-\mathrm{B}_{\mathrm{i}}\end{array}$ \\
\hline 6.418 & 11.454 & 5.036 & 0 & 8 & 0 & 4 & 0 & 20 \\
\hline 6.424 & 11.173 & 4.749 & 0 & 8 & 0 & 4 & 4 & 16 \\
\hline 6.617 & 8.830 & 2.213 & 0 & 0 & 0 & 0 & 8 & 32 \\
\hline 6.655 & 8.824 & 2.169 & 0 & 0 & 0 & 0 & 8 & 32 \\
\hline 6.758 & 9.895 & 3.137 & 0 & 4 & 0 & 0 & 4 & 32 \\
\hline 6.843 & 9.820 & 2.977 & 0 & 4 & 0 & 0 & 4 & 32 \\
\hline 7.107 & 10.607 & 3.500 & 4 & 4 & 0 & 0 & 4 & 28 \\
\hline 7.131 & 11.131 & 4.000 & 0 & 8 & 0 & 0 & 4 & 28 \\
\hline 7.168 & 11.287 & 4.119 & 0 & 0 & 0 & 4 & 8 & 36 \\
\hline 7.180 & 10.746 & 3.566 & 4 & 4 & 0 & 0 & 4 & 28 \\
\hline 7.517 & 11.110 & 3.593 & 0 & 8 & 0 & 0 & 4 & 28 \\
\hline 8.135 & 9.805 & 1.670 & 4 & 8 & 0 & 4 & 0 & 0 \\
\hline 8.143 & 9.717 & 1.574 & 4 & 8 & 0 & 4 & 0 & 0 \\
\hline
\end{tabular}

To understand the energy contribution of breaking chain, chain-icosahedra, inter-icosahedra and intra-icosahedra bonds in the rigid case (prior to relaxation) we applied a least squares fitting to the rigid separation energies for our entire combinatorial search data set (see Table III in the supplemental material). This set includes those surface structures that were neglected in Table I because they gave higher $\mathrm{W}_{\text {sep }}$ values at their spatial local. We use the entire data set in order to avoid bias that might result from using only surfaces giving the lowest $\mathrm{W}_{\text {sep }}$ values. Within the system of equations we have also added the cohesive energy constraint. We found the cohesive energy of $\mathrm{B}_{11} \mathrm{C}_{\mathrm{p}}(\mathrm{CBC})$ to be $-6.500 \mathrm{eV} /$ atom where the doublet and triplet atomic states were used for boron and carbon respectively. This value seems reasonable compared to work by Bylander et al. [23]. They find a cohesive energy of -7.299 eV/atom using Wigner correlation which is expected to be more LDA like. It is known that LDA overestimates cohesive energies compared to the PBE GGA functional which we use and compared to experiment [24] (note especially carbon diamond in Table 5 of Ref. 24). The result of our fit is shown in Table II and has a 0.15 coefficient of variation of the root mean square error. This simplified bond energy model demonstrates how the different bonds contribute to the rigid separation energy and indeed gives a bond energy ordering that matches the above mentioned bond stiffness ordering.

TABLE II. Rigid bond energies based on a least squared fit of all rigid surface structures explored in this study along with the cohesive energy constraint (see supplemental data).

\begin{tabular}{|c|c|}
\hline Bond type & Bond energy [-eV] \\
\hline Intra-chain & 7.11 \\
Chain-icosahedra & 4.43 \\
Inter-icosahedra & 4.31 \\
Intra-Icosahedra & 1.37 \\
\hline
\end{tabular}

Next the separated surfaces are allowed to fully relax. The surface relaxation energies are shown in column 3 of Table I and found to be substantial. It is helpful to look at a few specific examples of surface relaxation in boron carbide. The examples given in Fig. 2 represent a variety of cases, and give a more detailed sense of the atomic relaxations observed. Figure 2 
illustrates final, relaxed structures for separations progressing on planes running perpendicular to the page at locales (a), (b), and (c) as shown in the Fig. 1 bulk model. Figure 2a shows a low energy separation in which a single boron atom is removed from each surface icosahedron as well as broken inter-icosahedra and chain-icosahedra bonds; in this case significant relaxation occurs as seen both geometrically and in the relaxation energy. Figure $2 b$ shows an example of separation where the icosahedra are more completely cleaved, only intra-icosahedra bonds are broken; significant geometrical relaxation is observed. Figure $2 \mathrm{c}$ shows separation with the highest $W_{\text {sep }}$. Note that this separation passes through the chains, chain-icosahedra and intericosahedra bonds. While the chain center boron lies down, the icosahedra remain intact; this scenario results in the smallest of the relaxation energies observed.

One can see from Table I that there is not a correlation between the rigid separation energies and $W_{\text {sep. }}$. In fact, the smallest $W_{\text {sep }}=6.418 \mathrm{~J} / \mathrm{m}^{2}$ is associated with the largest rigid separation energy. This is because surface relaxation plays a substantial role in lowering the $W_{\text {sep }}$ and $\sigma$ of boron carbide. For example, in the case of the lowest $W_{\text {sep }}$ just mentioned, the magnitude of the surface relaxation is about $44 \%$ of the magnitude of the rigid separation energy. This demonstrates the necessity of accounting for surface relaxation in the boron carbide surface energy.

The separations that are least favorable are those that leave the icosahedra intact - see Fig. 2c and the last two rows of Table I. They are the least favorable because they have the highest separation energies $W_{\text {sep }}$. This seems reasonable since the highest energy bonds, the intra-chain and chain-icosahedra, are broken. However, a sizable majority of the separations have a higher rigid separation energy. Nevertheless, all of those higher rigid separations also had a higher surface relaxation energy than the intact icosahedra case. It is the low surface relaxation energy of the intact icosahedra surfaces that seems to make such separation motifs unfavorable.

The underlying order and structural units comprising solid boron carbide can shed more light on these results. It has been known for many years [25] that surface relaxation displacements in fcc metals are of the order of $0.1 \AA$ or less, which is roughly an order magnitude less than seen here. One must consider the relative openness of the boron carbide structure. The atomic packing of the fcc lattice is $74 \%$ of the cell volume, whereas the boron carbide atomic packing is only $32 \%$ of its cell volume, less than half of the fcc value. The open space is a consequence of the chain-icosahedra structural units from which the solid is comprised. This atomic openness is likely why surface relaxation is so large for boron carbide.

There is, however, a special case that needs to be considered. While boron carbide is not atomically close packed, it is close packed with respect to icosahedra packing - an important distinction. When icosahedra are intact, spatial constraints resist surface relaxation. Thus a lower surface relaxation is implied for separation paths that leave icosahedra intact. Such separation paths also involve breaking stiffer, more energetic bonds. Together, a low surface relaxation and high cost of breaking bonds conspire to make separations that leaves the icosahedra intact unfavorable.

\section{Comparison with Experiment}

Fracture surface energies have been measured for boron carbide by Hollenberg [26] and Walther. Boron Carbide specimens with controlled precracks were subjected to increasing loads until crack propagation occurred. This allows the critical stress intensity factor $K_{I C}$ to be measured. Then the fracture surface energy $\gamma_{f}$ is determined from 


$$
\gamma_{f}=\left[K_{I C}{ }^{2}\left(1-v^{2}\right)\right] / 2 E,
$$

where measured values were inserted for $K_{I C}$, Poisson's ratio $v$ and Young's modulus $E$. Hollenberg [26] and Walther report an average measured fracture surface energy $\gamma_{f}=3.27 \mathrm{~J} / \mathrm{m}^{2}$. This compares well with the surface energy value $\sigma=3.21 \mathrm{~J} / \mathrm{m}^{2}$ computed in this work.

The implications of this rather close agreement between the measured $\gamma_{f}$ and computed $\sigma$ require further discussion. The fracture surface energy as given by Eq. (2) is a measure of the fracture toughness [26-28]. As such, it includes energies expended by plastic flow, viscous flow, and nonrecoverable elastic strain. Moreover, the fracture path may well not be limited to a single plane, being influenced by, e.g., lattice trapping, grain boundaries, and other defects. Hollenberg [26] and Walther noted that the magnitude of their measured boron carbide fracture surface energies are near the anticipated thermodynamic free surface value, and concluded that fracture energies expended by plastic flow, viscous flow, and nonrecoverable elastic strain must be relatively small for boron carbide [29-31]. They also stated that scanning electron microscopy reveals almost featureless fracture surfaces for boron carbide, suggesting that fracture surface roughness effects are also small.

The DFT computations reported here assume planar separation, and ignore plastic flow, viscous flow, and nonrecoverable elastic strain. The close agreement between the DFT surface energy and the experimental fracture surface energies is consistent with Hollenberg [26] and Walther's conclusions regarding relatively small effects of fracture surface roughness, plastic flow, viscous flow, and nonrecoverable elastic strain in boron carbide fracture.

Surface energies of other low-index surface planes of boron carbide need to be computed to assess the degree of variation of surface energy with surface plane. This would be important information to form a more complete understanding of boron carbide surface energetics. In particular, it would be interesting to determine the lowest surface energy plane of boron carbide. That would provide more information for comparison with the fracture surface experiments of Ref. 25.

\section{Conclusions}

First-principles, density functional computations of the surface energy of the $\{10 \overline{1} 1\}$ plane of the $\mathrm{B}_{11} \mathrm{C}_{\mathrm{p}}(\mathrm{CBC})$ polytype of boron carbide have been carried out. The surface energy was found to be $3.21 \mathrm{~J} / \mathrm{m}^{2}$. Good agreement was found with the experimental average fracture surface energy as measured by Hollenberg [26] and Walther. This is consistent with their [26] assessment that energy losses due to plastic flow, viscous flow, nonrecoverable elastic strain, and surface roughness may be relatively small contributors to the fracture surface energy of boron carbide. Surface relaxation was found to be relatively large, lowering the surface energy by $44 \%$. The planar separation along $\{10 \overline{1} 1\}$ with the lowest surface energy has the highest surface relaxation. The large surface relaxation is likely due to the relatively open lattice structure of boron carbide. Intact icosahedra surfaces were associated with the highest $W_{\text {sep }}$ values and thus were expected to be unfavorable energetically. Surfaces with intact icosahedra are unlikely due to a reduced surface relaxation constrained by icosahedra close packing and strong interconnecting bonds. The rigid bond energy ordering of the bulk intra-chain, chain-icosahedra, 
inter-icosahedra, intra-icosahedra bonds was found to be in agreement with the stiffness ordering from experiment.

\section{Acknowledgements}

T. D. Beaudet thankfully acknowledges that this research was supported in part by a Postgraduate Research appointment to the U.S. Army Research Laboratory administered by the Oak Ridge Institute for Science and Education. J. R. Smith gratefully acknowledges partial support from the U.S. Army Research Laboratory. Useful comments from J. McCauley, J. LaSalvia, J. Swab, and K. Hemker are especially appreciated.

\section{References}

[1] H. Werheit, S. Shalamberidze, J. Phys.: Condens. Matter 24 (2012) 385406.

[2] K. M. Reddy, J. J. Guo, Y. Shinoda, T. Fujita, A. Hirata, J. P. Singh, J. W. McCauley, M. W. Chen, Nat. Commun. 3 (2012) 1052.

[3] F. Thevenot, J. Eur. Ceram. Soc., 6 (1990) 205.

[4] H. W. Kim, Y. H. Koh, H. E. Kim, J. Am. Ceram. Soc., 83 (2000) 2863.

[5] K. A. Schwetz, L. S. Sigl, L. Pfau, J. Solid State Chem., 133 (1997) 68.

[6] L. S. Sigl, J. Eur. Ceram. Soc., 18 (1998) 1521.

[7] Z. Zahariev, D. Radev, J. Mater. Sci. Lett. 7 (1998) 695.

[8] V. J. Skorokhod, M. D. Vlajic, V. D. Krstic, J. Mater. Sci. Lett. 15 (1996) 1337.

[9] H. Lee, R. F. Speyer, J. Am. Ceram. Soc. 85 (2002) 1291.

[10] C. Kaufmann, D. Cronin, M. Worswick, G. Pageau, A. Beth, Shock Vib. 10 (2003) 51.

[11] P. Hohenberg, W. Kohn, Phys. Rev. B 136 (1964) 864.

[12] W. Kohn, L. J. Sham, Phys. Rev. A 140 (1965) 1133.

[13] V. Domnich, S. Reynaud, R. A. Haber, M. Chhowalla, J. Am. Ceram. Soc. 94 (2011) 3605.

[14] D. E. Taylor, J. W. McCauley, T. W. Wright, J. Phys.: Condens. Matter 24 (2012) 505402.

[15] J. P. Perdew, K. Burke, M. Ernzerhof, Phys. Rev. Lett. 77 (1996) 3865.

[16] J. P. Perdew, K. Burke, M. Ernzerhof, Phys. Rev. Lett. 78 (1997) 1396.

[17] CP2K Open Source Molecular Dynamics, http://www.cp2k.org/.

[18] J. VandeVondele, M. Krack, F. Mohamed, M. Parrinello, T. Chassaing, J.Hutter, Comput. Phys. Commun. 167 (2005) 103.

[19] S. Goedecker, M. Teter, J. Hutter, Phys. Rev. B 54 (1996) 1703.

[20] C. Hartwigsen, S. Goedecker, J. Hutter, Phys. Rev. B 58 (1998) 3641.

[21] M. Krack, Theor. Chem. Acc. 114 (2005) 145.

[22] D. Gosset and M. Colin, J. Nucl. Mater. 183 (1991) 161.

[23] D. M. Bylander, L. Kleinman, S. Lee, Phys. Rev. B 42 (1990) 1394.

[24] I. Lee, R. M. Martin, Phys. Rev. B 56 (1997) 7197.

[25] J. R. Smith, A. Banerjee, Phys. Rev. B 37 (1988) 10411.

[26] G. Hollenberg and G. Walther, J. Am. Ceram. Soc. 63 (1980) 610.

[27] Y. Wei, J. W. Hutchinson, Int. J. Fracture 95 (1999) 1.

[28] Y. Jiang, Y. Wei, J. R. Smith, J. Hutchinson, A. G. Evans, Int. J. Mater. Res. 10 (2010) 1.

[29] Shock-induced amorphization has been seen in boron carbide. Nanoscale amorphous bands have been reported by Chen [30] et al. (see also [31]) for particles ejected from craters in boron carbide produced by projectile impacts when projectile speeds are higher than $850 \mathrm{~m} / \mathrm{s}$. These 
high-speed impact particles are expected to be rather different from the crack surfaces found in the Hollenberg [26] and Walther experiments, however. Cracks seen propagating radially from the impact craters of Ref. 30 may be well represented by the experiments of [26] however. [30] M. Chen, J. W. McCauley, K. J. Hemker, Science 299 (2003) 1563. [31] Q. An. A. J. Goddard III, T. Cheng, Phys. Rev. Lett. 113 (2014) 095501. 


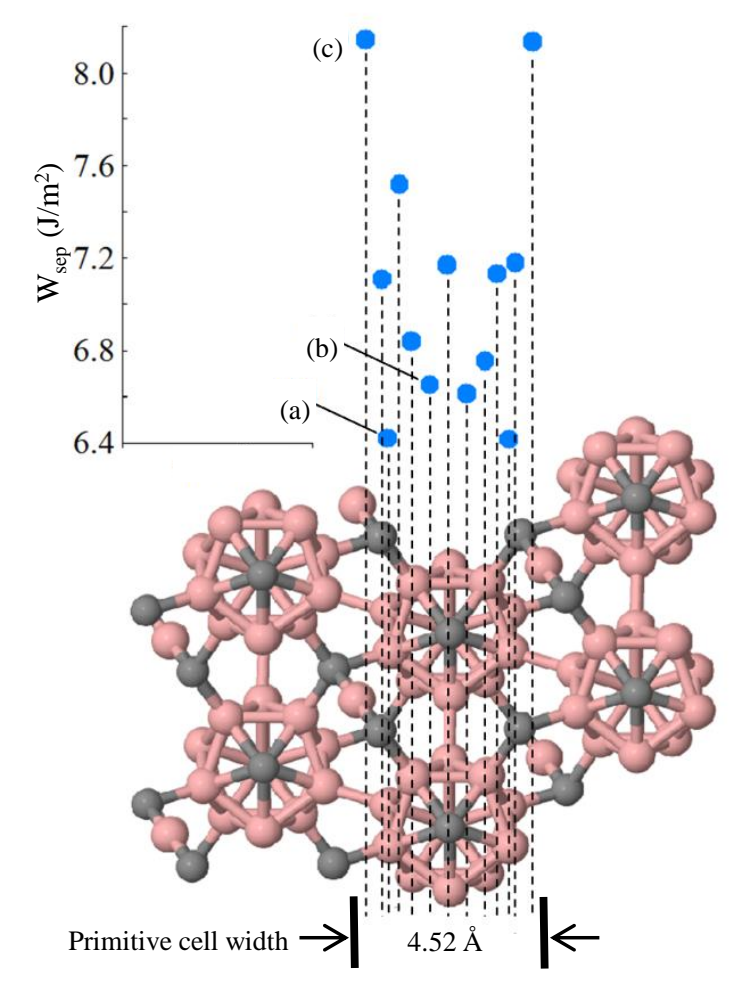

Fig 1: Beaudet, Smith and Adams

Figure 1

Fig 1: Beaudet, Smith and Adams

Figure 1

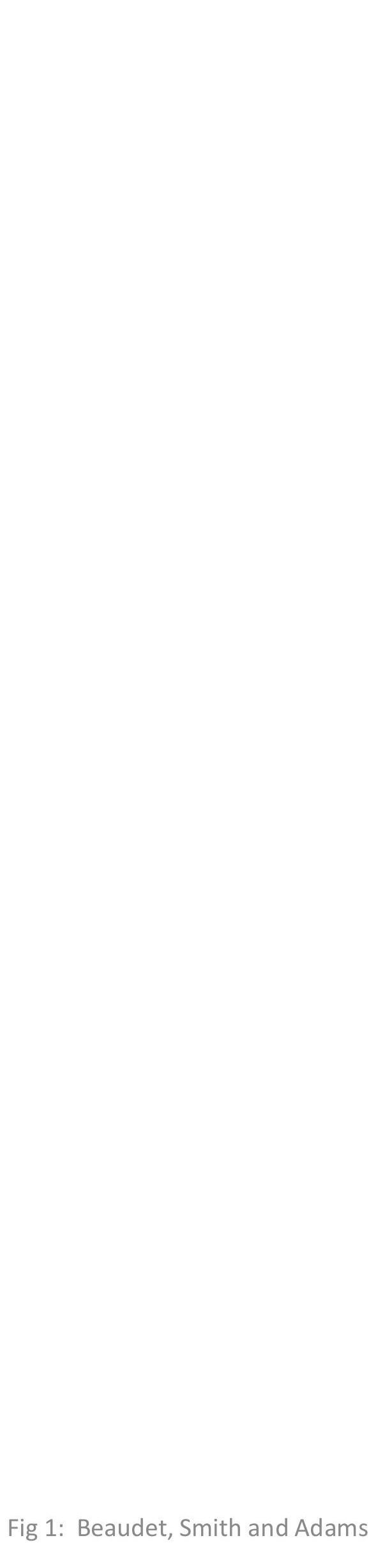

'

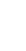

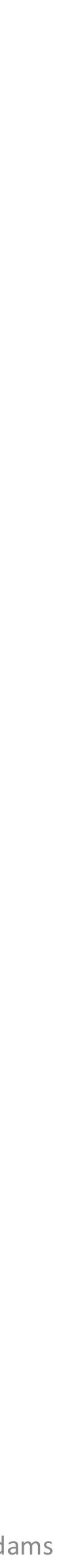



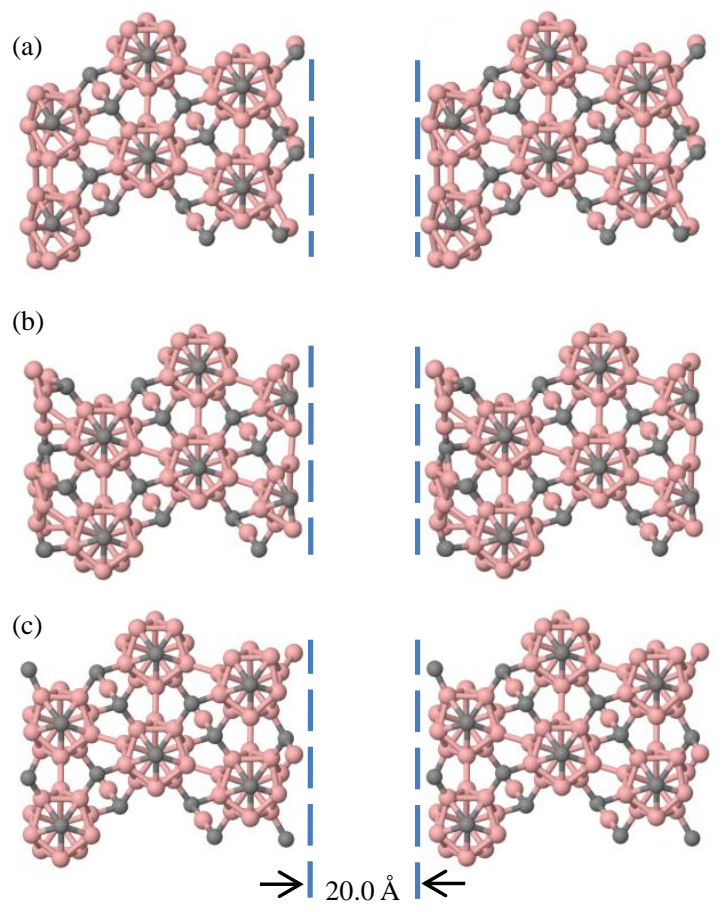

Interfacial separation 
FIG. 1. Plot of the lowest $B_{11} C_{p}(C B C)$ works of separation for the $\{10 \overline{1} 1\}$ family of planes. Each dashed line corresponds to a possible separation plane (perpendicular to the page) that passes through the solid. The $W_{\text {sep }}$ values shown are the lowest found for each respective plane. Points labeled "a, b and c" correspond to the surface structures shown in Fig. 2.

FIG. 2. Separated $B_{11} C_{p}(C B C)$ surfaces. Subfigures a) to c) correspond to the labeled separations shown in Fig. 1. Here, the surface relaxation can be seen structurally for each case. The separation is indicated by the blue dotted lines. 